\title{
A shift in the classic management of parotidectomy - the facelift approach
}

\author{
Mihai-Liviu Ciofu', Mihail Dan Cobzeanu², Otilia Boisteanu', Daniela Sulea', Razvan Giuvara', \\ Alexandra Carp' ', Eugenia Popescu', Victor-Vlad Costan'1 \\ ${ }^{1}$ Faculty of Dental Medicine, Oral and Maxillofacial Surgery, "Grigore T. Popa” University of Medicine and \\ Pharmacy, Iasi, Romania \\ ${ }^{2}$ Faculty of Medicine, Otolaryngology, "Grigore T. Popa” University of Medicine and Pharmacy, Iasi, Romania
}

\begin{abstract}
BACKGROUND. Improving the aesthetic and functional outcomes of parotidectomy is a constant concern of recent studies. We describe our experience on this topic by employing techniques inspired from rhytidectomy.

MATERIAL AND METHODS. We performed a retrospective study including patients that underwent parotidectomy through a facelift approach and we documented the details of the technique and the postoperative care and outcomes.

RESULTS. A total of 247 patients with parotid gland tumors were operated using the facelift approach. A superficial or quasi-total parotidectomy was performed, followed by a superficial musculoaponeurotic system-plasty. The postoperative outcomes were favourable regarding both the appearance and function, with minimal complications. The advantages of the employed technique are discussed.

CONCLUSION. It is possible to decrease the sequelae, simplify the postoperative care and improve the aesthetic outcome of parotidectomy cases by employing the facelift approach and the superficial musculoaponeurotic system (SMAS) reconstruction of the post-parotidectomy defect.
\end{abstract}

KEYWORDS: parotidectomy, parotid tumor, facelift, facial nerve, incision, SMAS.

\section{INTRODUCTION}

Classic approaches to performing parotidectomy have proven their usefulness over the course of time. Nevertheless, the presence of scars in the cervical region can be quite obvious and even cause pain or minor movement restriction, particularly if myoplasty was additionally performed using a pedicled cervical muscle flap, like the frequently employed sternocleidomastoid (SCM) or digastric flaps ${ }^{1}$. The current trend is to minimize the sequelae of parotid surgery, especially in cases of benign tumors. Multiple variants of incisions have been imagined to overcome the previously described disadvantages of classic approaches ${ }^{2-4}$. In addition, more emphasis is given to the volumetric reconstruction of the post-parotidectomy volume deficit and the prophylaxis of Frey's syndrome $^{5}$. Authors have different preferences regarding the techniques employed in parotid gland surgery.
The aim of this manuscript is to present our choice of access and volume reconstruction techniques by using the facelift incision and the superficial musculoaponeurotic system - plasty (SMAS - plasty). We outline the positive aspects of using an approach inspired from aesthetic procedures for the safe removal of benign parotid gland tumors, in an attempt to improve the appearance, simplify the postoperative care and also reduce the occurrence of the sequelae associated with this type of surgery.

\section{MATERIAL AND METHODS}

We conducted a retrospective clinical study by reviewing the medical charts of patients undergoing parotidectomy via the facelift incision. We only included adult patients with a minimum follow-up time of six months. Patients with previous surgery in the parotid region were 


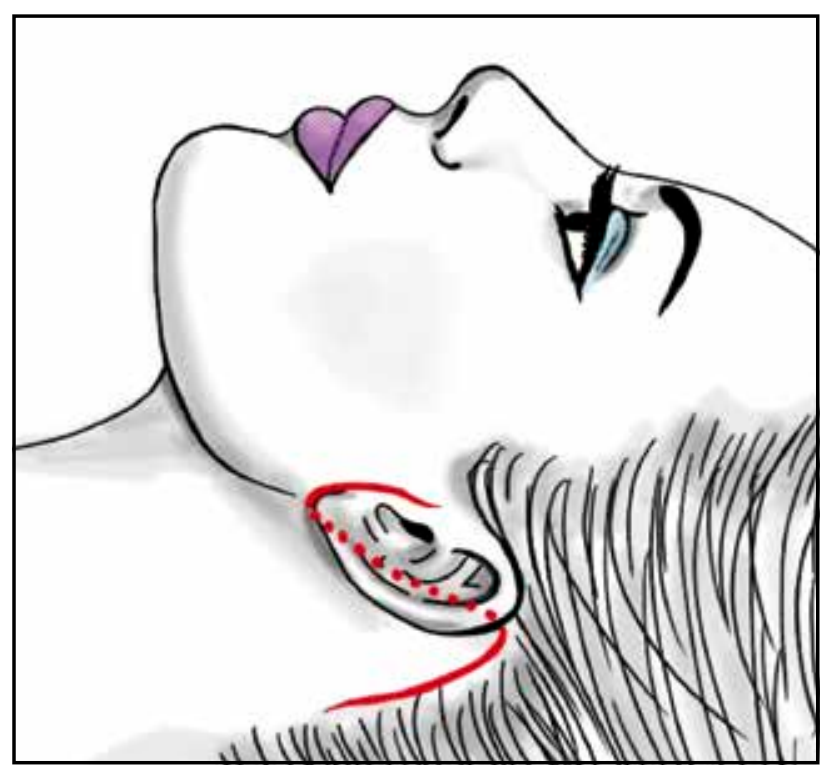

Figure 1. Outline of a facelift type of skin incision.

excluded from the study. The general information, as well as data regarding the surgical access, technique and postoperative results were all noted.

\section{RESULTS}

\section{Patients and procedures}

In total, 247 patients were identified, 135 men and 112 women, with ages between 8 and 79 years old. All patients underwent surgery for the removal of parotid gland tu-

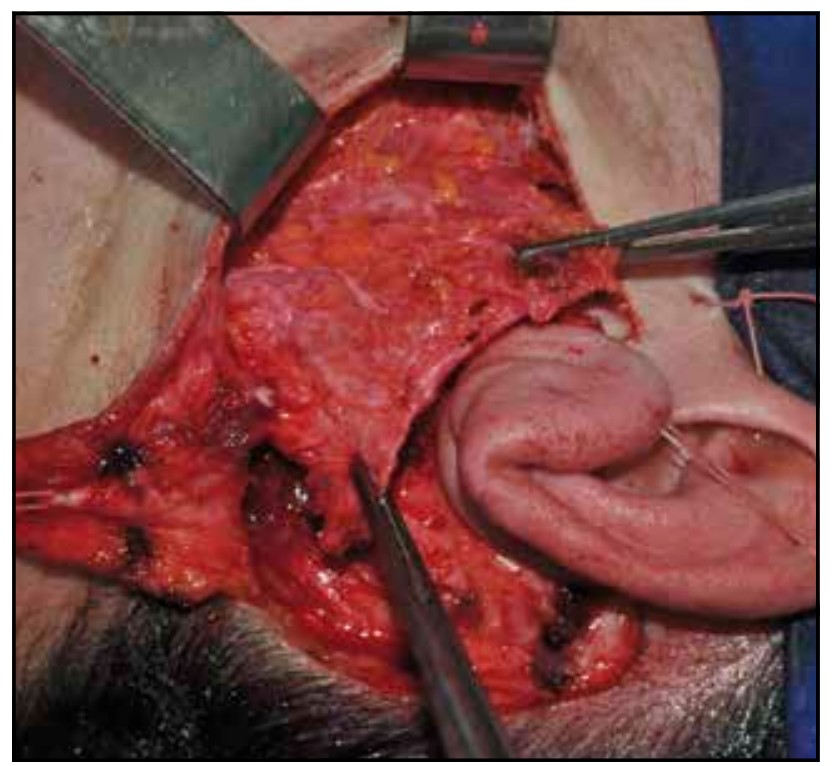

Figure 2. The facelift approach to parotidectomy. Intraoperative view of the undermined skin flap and of the dissected SMAS flap. mors with clinical and paraclinical characteristics of benign lesions. The procedures were performed under general anaesthesia in 236 cases and under local anaesthesia (with intravenous sedation) in 11 patients. There were 211 superficial parotidectomies and 36 quasi-total parotidectomies performed. The facial nerve was preserved in all included cases by conducting an anterograde dissection in 209 cases and by a retrograde approach in 38 patients. An associated plasty of the volume deficit was performed using the superficial musculoaponeurotic system (SMAS) in all patients.

\section{Surgical technique}

Hydro-dissection was performed as an initial step, using a mixture of local anaesthetic with vasoconstrictor and saline, injected over the entire dissection area, to facilitate tissue separation, particularly in the thinner mastoid region. A facelift type of incision was used for access. The incision line was hidden in the natural skin creases in front of and behind the ear, turning superiorly in the mastoid area to then run obliquely adjacent to the hairline (Figure 1).

Superficial dissection was then performed in the subcutaneous plane, followed by separation of the SMAS flap from the parotid-masseteric fascia. The principal landmarks for identifying the main trunk of the facial nerve were the tip of the tragal cartilage, the posterior belly of the digastric muscle and the stylomastoid artery announcing the nerve trunk found below. Following tumor removal by parotidectomy, the exposed facial nerve branches were covered using the SMAS flap, which was folded into the defect and sutured, in order to render volume to the region.

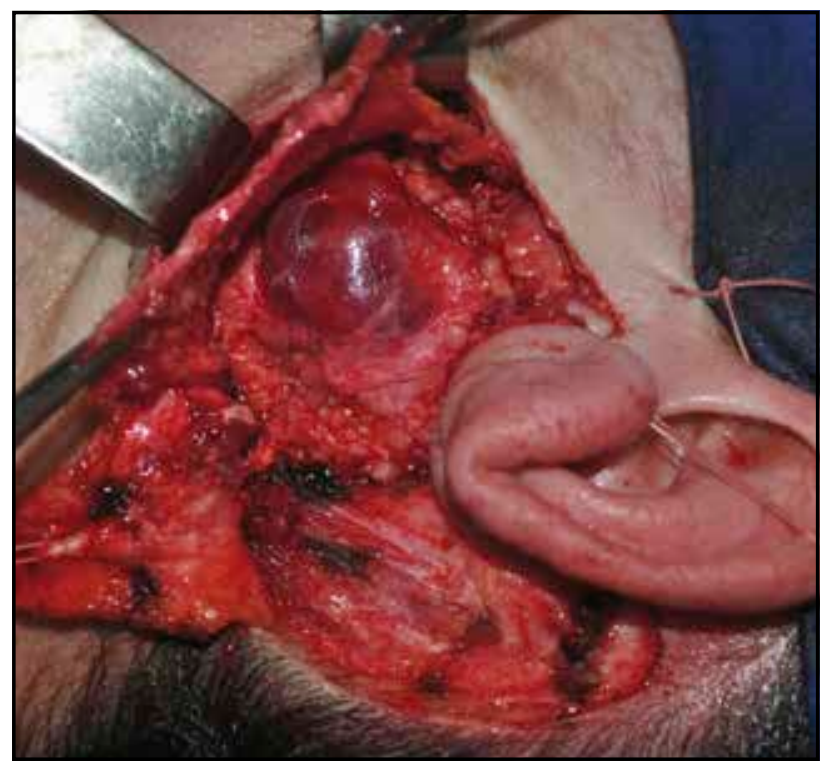

Figure 3. Intraoperative view of the benign parotid gland tumor situated in the superficial lobe. The image demonstrates adequate exposure of the entire parotid region. 


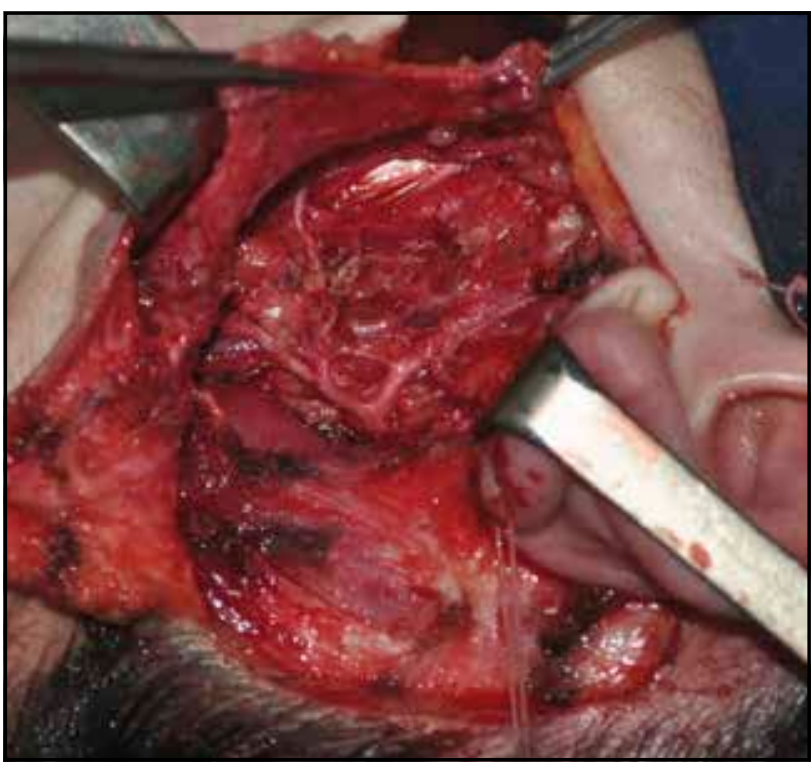

Figure 4. Surgical field after the removal of the parotid tumor by superficial parotidectomy with facial nerve preservation. View of the facial nerve branches overlying the retromandibular vein, the upper insertion of the SCM and the posterior belly of the digastric muscle

To illustrate the surgical technique, we present intraoperative images of a case involving a facelift type of access performed for the removal of a benign parotid gland tumor (pleomorphic adenoma) by superficial parotidectomy with facial nerve preservation followed by plasty using the SMAS (Figures 2-7).

\section{Postoperative care and outcomes}

A modelling compressive dressing was applied at the end of the surgery and maintained in between one and three days after the procedure.

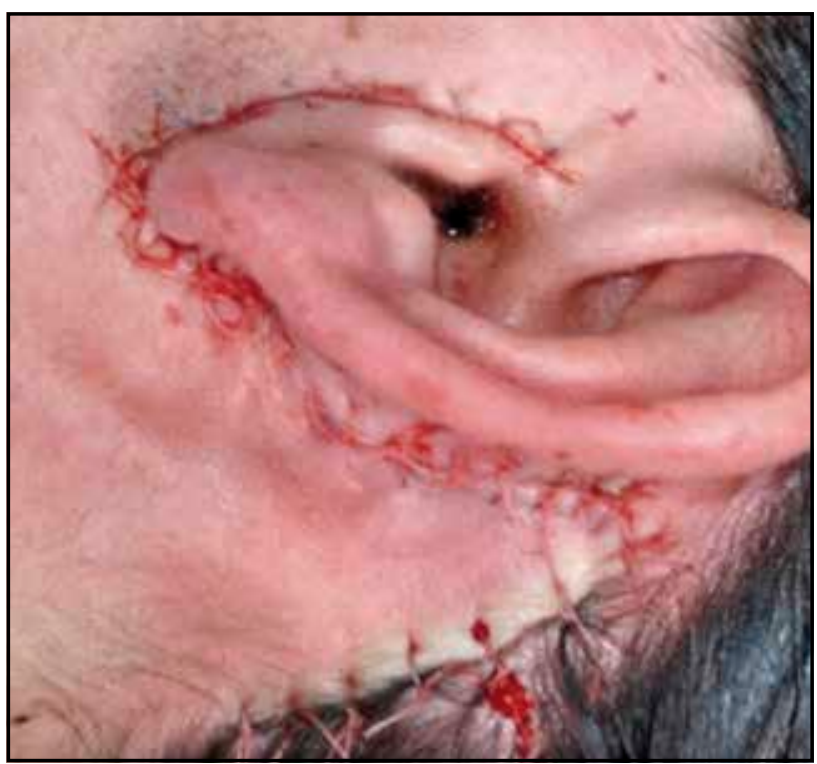

Figure 6. Immediate postoperative aspect following wound closure.

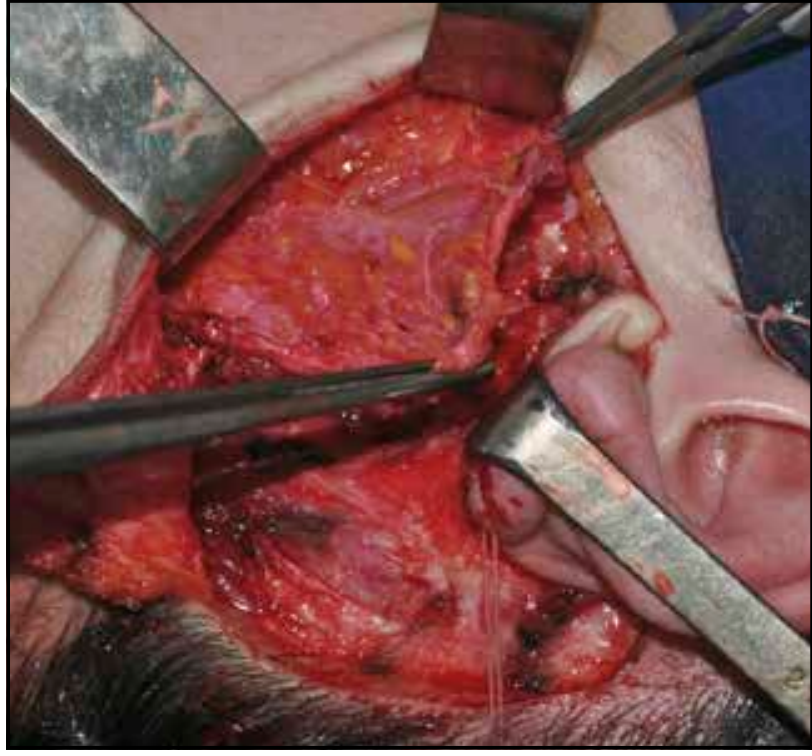

Figure 5. Intraoperative view of the SMAS- plasty. The SMAS flap is used to cover the exposed facial nerve branches and the remaining deep parotid tissue, as well as for filling the soft tissue defect..

Ecchymosis and postoperative edema were the most frequent complications. There were three cases of hematomas that necessitated drainage, without any further complications after treatment. There were no occurrences of skin flap necrosis. No cases of wound infections were noted.

A transitory facial nerve paralysis was present in all patients immediately after surgery, but gradually disappeared within three months with resolution of normal function. Facial symmetry was accurately restored in all cases of superficial parotidectomy. A minor asymmetry due to a

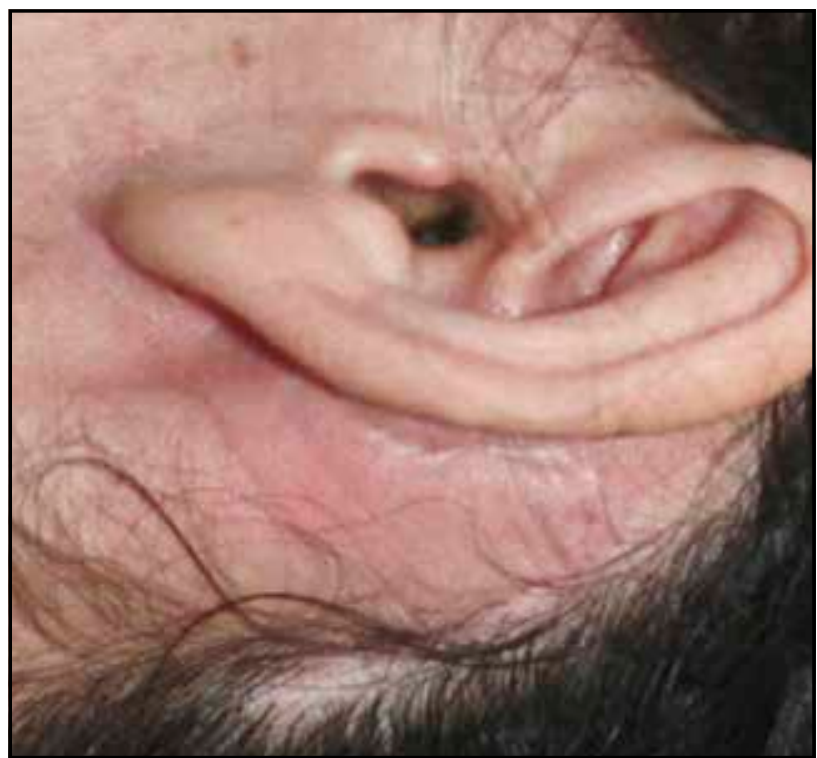

Figure 7. View of the postoperative scar one month after surgery. 
slightly depressed parotid region was noticed in the patients that underwent quasi-total parotidectomy at one year after surgery. Autologous fat grafting was performed in 6 of those patients. The postoperative scars were barely noticeable in the majority of cases. In 37 patients a slightly stretched scar developed in the mastoid region but was well camouflaged by the surrounding hair. There were 4 cases of Frey syndrome that were well managed by Botulinum toxin injections. No salivary fistula formation was encountered during the follow-up period.

\section{DISCUSSIONS}

The most obvious advantage of the facelift approach to parotidectomy is aesthetic, since the incision lines are well hidden in the normal periauricular skin creases and camouflaged by the hairline in the mastoid region, particularly in individuals wearing long hair. Shaving of the parotid region is not necessary, which contributes to the rapid work and social reinsertion of patients undergoing parotidectomy. The incisions that follow the natural skin creases generally result in inconspicuous scars. The positioning of the ear lobe at the end of surgery is important, in order to avoid increased traction and the appearance of "pixie ear". The area overlying the mastoid is where stretched scars have been encountered in 37 of the patients included in our study. Similar outcomes are reported by other authors ${ }^{7,8}$ and are attributed to the more deficient vascularity of this thin, adherent skin surface and the relatively higher tension on the sutures placed at the superior aspect of the skin flap in the mastoid region, particularly in cases of important edema or hematoma development. Still, even in the rare event of un-aesthetic scars formation, they are well hidden by the auricle and the adjacent hair.

From our experience, the structures that can be easily accessed with the usual pattern of facelift incision are the entire parotid bed, including the tail of the gland, the superior aspect of the sternocleidomastoid muscle and the posterior belly of the digastric muscle. Additionally, the SMAS can be readily identified and lifted from the parotid-masseteric fascia, forming a flap that serves reconstructive purposes. We could access both the superficial and deep lobes of the parotid gland by using the facelift approach. Similar findings were reported by other authors, stating that the Blair type of incision and the facelift incision offer analogous access and visibility for both superficial and quasi-total parotidectomies ${ }^{9}$.

Concerns regarding the suitability of this type of access have been raised in relation to malignant parotid gland tumors, recurrent tumors, extremely large benign tumors, tumors extended towards the latero-pharynx, as well as large tumors located antero-inferiorly ${ }^{1,7}$. Additionally, relative contraindications to this technique are all systemic conditions that influence vascularization and wound healing, due to possible skin flap necrosis, although this is an extremely rare event, as reported in the existing literature ${ }^{7}$. In our case series, there were no cases of skin flap necrosis.

In our view, the facelift approach offers sufficient exposure for an adequate removal of the majority of parotid gland tumors exhibiting initial clinical and paraclinical signs of benign lesions. Modifications of the incision line are possible in order to increase the surgical access in selected cases, towards the zygomatic and temporal regions, as well as towards the submandibular region and upper laterocervical region ${ }^{10}$. We do not commonly use the facelift approach for the removal of parotid tumors exhibiting signs of malignancy, due to concerns regarding access for neck dissection, for good local control of the tumor resection and the frequent need to also excise part of the SMAS. Other authors describe malignant parotid tumor removal using the facelift approach, with or without modifications, in cases in which the oncologic safety can be ensured ${ }^{10-a 12}$.

Following parotidectomy, a volume loss is noticeable in the parotid region, unless a reconstructive technique is employed. Classic methods of filling the retromandibular depression involve myoplasty using the digastric or SCM flaps ${ }^{1}$. We achieved good outcomes regarding both the appearance and function by performing a SMASplasty. Similar results are reported by other authors underlining the multiple benefits of this reconstructive method $^{2,3,13,14}$.

In patients with benign parotid gland tumors, there is usually excess SMAS, just like there is excess skin overlying the deformation in the parotid region. This surplus of SMAS provides an excellent filling material that compensates for the removed parotid tissue. Additionally, a small quantity of blood remaining under the SMAS flap is considered beneficial for restoring the volume of the region following fibrous changes. The importance of the sub-SMAS hematoma was also outlined by other authors ${ }^{13,15}$. When considered necessary, an associated reconstructive method can be used, utilizing the SMAS flap together with a SCM or digastric muscle flap. Another method that has been described for defect filling at the time of parotidectomy involves the use of a temporo-parietal fascial flap ${ }^{1,5}$. Additionally, structural fat grafting may be performed after quasi-total parotidectomy in order to ensure defect filling and restore symmetry, as performed in 6 of our patients in which SMAS-plasty did not provide stable volume one year after surgery.

The folded SMAS provides coverage and protection for the exposed facial nerve and acts like a barrier between the remaining glandular tissue and the overlying skin, thus preventing the occurrence of Frey syndrome. Many authors describe a significant decrease in the onset of gustatory sweating with the use of the SMAS-plasty ${ }^{7,14,16}$. In our case series, there were only 4 cases of Frey syndrome onset after parotidectomy. We consider that the 
integrity of the SMAS flap helps prevent the onset of the syndrome, since any piercing holes in the surface of the flap may allow the direct connection between the parasympathetic nerve fibers and skin.

Filling the post-parotidectomy volume deficit by using the SMAS flap does not imply any functional changes and therefore decreases the local morbidity associated with the use of pedicled muscle flaps, resulting in less bleeding, pain and edema, just like outlined in the results of our study. Thus, the drainage is not always necessary and compressive dressings are not generally required beyond three days after surgery, resulting in overall shortened hospital stay. Nevertheless, judicious haemostasis must be ensured at the end of the procedure, in order to prevent postoperative bleeding and ensure favourable healing. Decreased complication rates are also described in other studies in relation to using the SMAS flap for parotidectomy defects ${ }^{7}$.

\section{CONCLUSIONS}

In our experience, the facelift approach proved suitable for the removal of parotid gland tumors with benign attributes, ensuring sufficient access, visibility and the possibility to perform a SMAS-plasty, which further ensured favourable aesthetic and functional outcomes, with a low morbidity and simplified postoperative care.

Acknowledgements: The authors would like to thank all the medical personnel that contributed to the present study.

Conflict of interest: The authors have no conflict of interest to disclose.

Contribution of authors: All authors have equally contributed to this work.

\section{REFERENCES}

1. Irvine LE, Larian B, Azizzadeh B. Locoregional parotid reconstruction. Otolaryngol Clin North Am. 2016;49(2):435-46. DOI: 10.1016/j.otc.2015.10.012. Epub 2016 Feb 16.

2. de Vicente JC, González-García M, de Villalaín L, Fernández-Valle Á. Modified facelift approach combined with a superficial musculoaponeurotic system flap in the treatment of benign parotid tumors. J Craniomaxillofac Surg. 2015;43(8):1655-61. DOI: 10.1016/j.jcms.2015.06.049. Epub 2015 Jul 10.
3. Bianchi B, Ferri A, Ferrari S, Copelli C, Sesenna E. Improving esthetic results in benign parotid surgery: statistical evaluation of facelift approach, sternocleidomastoid flap, and superficial musculoaponeurotic system flap application. J Oral Maxillofac Surg. 2011;69(4):1235-41. DOI: 10.1016/j. jons.2010.03.005. Epub 2010 Aug 12.

4. Zhang J, Jiang Q, Na S, Pan S, Cao Z, Qiu J. Minimal scar dissection for partial parotidectomy via a modified cosmetic incision and an advanced wound closure method. J Oral Maxillofac Surg. 2019;77(6):1317.e1-1317.e9. DOI: 10.1016/j.joms.2019.02.036. Epub 2019 Mar 2.

5. Movassaghi K, Lewis M, Shahzad F, May JWJr. Optimizing the aesthetic result of parotidectomy with a facelift incision and temporoparietal fascia flap. Plast Reconstr Surg Glob Open. 2019;7(2):e2067. DOI: 10.1097/ GOX.0000000000002067. eCollection 2019 Feb.

6. Upile T, Jerjes WK, Nouraei SA, Grant W, Singh S, Sudhoff H, et al. Further anatomical approaches to parotid surgery. Eur Arch Otorhinolaryngol. 2010;267(5):793-800. DOI: 10.1007/s00405-009-1148-8. Epub 2009 Dec 1.

7. Grover N, D'Souza A. Facelift approach for parotidectomy: an evolving aesthetic technique. Otolaryngol Head Neck Surg. 2013;148(4):548-56. DOI: 10.1177/0194599812475221. Epub 2013 Feb 4.

8. Griffin JE, Jo C. Complications after superficial plane cervicofacial rhytidectomy: a retrospective analysis of 178 consecutive facelifts and review of the literature. J Oral Maxillofac Surg. 2007;65(11):2227-34.

9. Nouraei SA, Al-Yaghchi C, Ahmed J, Kirkpatrick N, Mansuri S, Singh A, et al. An anatomical comparison of Blair and facelift incisions for parotid surgery. Clin Otolaryngol. 2006;31(6):531-4.

10. Giotakis EI, Giotakis AI. Modified facelift incision and superficial musculoaponeurotic system flap in parotid malignancy: a retrospective study and review of the literature. World J Surg Oncol. 2020;18(1):8. DOI: 10.1186/ s12957-020-1785-3.

11. Honig JF. Facelift approach with a hybrid SMAS rotation flap advancement flap in parotidectomy for prevention of scars and contour deficiency affecting the neck and sweat secretion of the cheek. J Craniofac Surg. 2004;15 (5):797-803

12. Foustanos A, Zavrides H. Face-lift approach combined with a superficial musculoaponeurotic system advancement flap in parotidectomy. Br J Oral Maxillofac Surg. 2007;45(8):652-5.

13. Kim IK, Cho HW, Cho HY, Seo JH, Lee DH, Park SH. Facelift incision and superficial musculoaponeurotic system advancement in parotidectomy: case reports. Maxillofac Plast Reconstr Surg. 2015;37(1):40. DOI: 10.1186/ s40902-015-0040-2.

14. Dell'Aversana Orabona G, Salzano G, Abbate V, Piombino P, Astarita F, Iaconetta $G$, et al. Use of the SMAS flap for reconstruction of the parotid lodge. Acta Otorhinolaryngol Ital. 2015;35(6):406-11. DOI: 10.14639/0392100X-395.

15. Meningaud JP, Bertolus C, Bertrand JC. Parotidectomy: assessment of a surgical technique including facelift incision and SMAS advancement. J Craniomaxillofac Surg. 2006;34(1):34-7.

16. Kyrgidis A, Triaridis S. Facelift approach in benign parotid surgery, Frey syndrome, and total superficial parotidectomy. J Oral Maxillofac Surg. 2011;69(10):2486-7. DOI: 10.1016/j.joms.2011.06.003. 